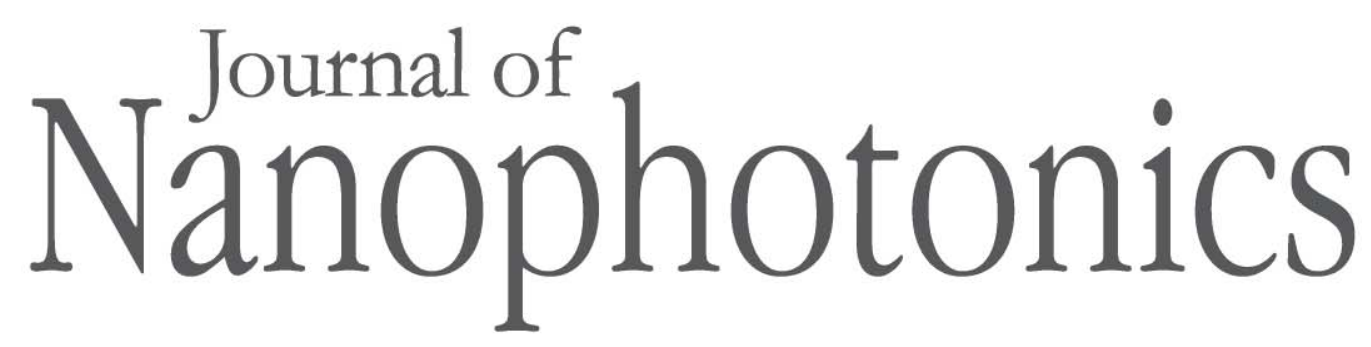

SPIEDigitalLibrary.org/jnp

\title{
Tuning the effective dielectric function of thin film metal-dielectric composites by controlling the deposition temperature
}

Jordi Sancho-Parramon

Vesna Janicki

Hrvoje Zorc 


\title{
Tuning the effective dielectric function of thin film metal-dielectric composites by controlling the deposition temperature
}

\author{
Jordi Sancho-Parramon, Vesna Janicki, and Hrvoje Zorc \\ Ruđer Bošković Institute, Bijenička cesta 54, Zagreb, 10000, Croatia \\ jsancho@irb.hr
}

\begin{abstract}
The influence of the substrate temperature on the effective optical behavior of $\mathrm{Ag}-\mathrm{SiO}_{2}$ composites obtained by electron beam evaporation was studied. Optical characterization of the composites was performed by means of spectroscopic ellipsometry measurements. The effective dielectric function of the composites, modeled using a multiple oscillator approach, could be widely tuned by controlling the deposition temperature. The spectral dependence of the composite absorption appeared to be better described with a Gaussian line shape than with the classical Lorentz oscillator model. The description of the effective dielectric function using standard effective medium theories failed and the experimental results could be explained only in the general framework of the Bergman spectral density theory. @ 2011 Society of Photo-Optical Instrumentation Engineers (SPIE). [DOI: 10.1117/1.3590238]
\end{abstract}

Keywords: metal-dielectric composites; metal island films; surface plasmon resonance; optical constants; effective medium theory; spectroscopic ellipsometry.

Paper 11015SSR received Jan. 28, 2011; revised manuscript received Apr. 15, 2011; accepted for publication Apr. 15, 2011; published online May 13, 2011.

\section{Introduction}

Metal-dielectric composites have been in the focus of interest due to the surface plasmon resonance phenomena and its large number of related applications, ranging from biological and chemical sensing to telecommunications. ${ }^{1}$ Recently, novel photonic applications of metaldielectric composites have been devised, in fields such as metamaterials operating in the optical range. ${ }^{2}$ The incorporation of metal-dielectric composites in multilayer structures enables combining the surface plasmon resonance and the interference effects that take place in the multilayer. Applications resulting from such combination are highly absorbing optical coatings, ${ }^{3,4}$ high luminosity reflectors,${ }^{5}$ and three-dimensional photonic structures. ${ }^{6,7}$ In order to design multilayer structures that include metal-dielectric composites it is required to have an accurate knowledge of the effective dielectric function of the composite. From the application point of view, it is desirable that this effective dielectric function can be tailored as widely as possible.

In the present work we study the effective dielectric function of $\mathrm{Ag}-\mathrm{SiO}_{2}$ composites in thin film phase. The effective dielectric function of the composites is determined from spectroscopic ellipsometry measurements, using a multiple oscillator model. It is shown that by varying the substrate temperature only, the effective dielectric function of the composites can be widely modified: composites deposited on heated substrates are characterized by a narrow surface plasmon resonance, while those deposited at room temperature show broader resonance and additional absorption in the infrared. It is shown that the obtained effective dielectric function cannot be described with classical effective medium theories. The experimental results can be explained only in the more general framework of the Bergman spectral density theory. The paper is structured as follows: Section 2 describes the theoretical models used for describing the

$1934-2608 / 2011 / \$ 25.00$ @ 2011 SPIE 
effective dielectric function of the composites. Section 3 presents the experimental details and in Sec. 4 the results of optical characterization of the samples are presented and discussed.

\section{Theory}

In order to describe the spectral dependence of the effective optical constants of a metal-dielectric composite, two different approaches are employed: i. using a parametric formula that accounts for the dispersion of the effective optical constants or ii. using an effective medium theory and computing the effective optical constants in terms of the optical constants of the composing metal and dielectric.

\subsection{Dispersion Parametric Formula: Oscillator Model}

The effective optical constants of metal-dielectric composites are basically characterized by a strong absorption peak around the central plasmon resonance wavelength. In the ideal case of spherical noninteracting particles one can assume that the dipolar contribution of the Mie theory dominates the extinction properties of the composite ${ }^{8}$ If the dispersion of the optical constants of metal follows the Drude model, the absorption has a Lorentzian line shape. ${ }^{9}$ Therefore, a commonly used dispersion model that accounts for the dispersion of the dielectric function of metal-dielectric composites, ${ }^{10,11}$ is the classical Lorentz oscillator:

$$
\varepsilon_{\text {Lorentz }}(E)=\frac{A_{n} B_{n} E_{n}}{E_{n}^{2}-E^{2}-i B_{n} E},
$$

where $A_{n}, B_{n}$ and $E_{n}$ are the strength, width, and central energy of the oscillator and $E$ is the photon energy. For random metal-dielectric composites, the particles present a size and shape distribution and one may expect that the absorption is due to a distribution of resonances rather than to a single resonance. Assuming a Gaussian distribution of resonances and that the width of this distribution is large enough, the imaginary part of the dielectric function may have a Gaussian line shape. ${ }^{12}$

$$
\begin{aligned}
& \varepsilon_{\text {Gauss }}(E)=\varepsilon_{G 1}(E)+i \varepsilon_{G 2}(E) \\
& \varepsilon_{G 2}(E)=A_{n} \mathrm{e}^{-\left[\left(E-E_{n}\right) / B_{n}\right]^{2}}-A_{n} \mathrm{e}^{-\left[\left(E+E_{n}\right) / B_{n}\right]^{2}}, \quad \varepsilon_{G 1}(E)=\frac{2}{\pi} P \int_{0}^{\infty} \frac{\xi \varepsilon_{G 2}(\xi)}{\xi^{2}-E^{2}} d \xi,
\end{aligned}
$$

where $\varepsilon_{G 1}$ and $\varepsilon_{G 2}$ are the real and imaginary part of the dielectric function and $P$ is the Cauchy principal value of the integral. In a general case, it appears that more than one oscillator might be necessary to account for the effective optical constants of metal-dielectric composites, due to inhomogeneous line broadening and percolation among particles. ${ }^{10}$ In this case, the effective optical constants are given by a multiple oscillator model, i.e., by the sum of several oscillators centered at different photon energies.

\subsection{Effective Medium Theories}

The second approach for describing the dispersion of the optical constants of composites uses effective medium theories. ${ }^{13}$ When the composite corresponds to a matrix with isolated and poorly interacting inclusions, the Maxwell-Garnett theory is often employed: ${ }^{13}$

$$
\frac{\varepsilon_{\mathrm{eff}}-\varepsilon_{m}}{\varepsilon_{\mathrm{eff}}+2 \varepsilon_{m}}=p \frac{\varepsilon_{i}-\varepsilon_{m}}{\varepsilon_{i}+2 \varepsilon_{m}}
$$


with $\varepsilon_{\text {eff }}$ being the effective dielectric function, $\varepsilon_{m}$ and $\varepsilon_{i}$ the dielectric functions of the matrix and of the inclusions, and $p$ the volume filling fraction of inclusions in the composite. If the composite arrangement is such that there is some degree of interconnection between components and the role of matrix and inclusions cannot be clearly stated, the Bruggeman expression ${ }^{13}$ appears to be more appropriate:

$$
p \frac{\varepsilon_{i}-\varepsilon_{\mathrm{eff}}}{\varepsilon_{i}+2 \varepsilon_{\mathrm{eff}}}+(1-p) \frac{\varepsilon_{m}-\varepsilon_{\mathrm{eff}}}{\varepsilon_{m}+2 \varepsilon_{\mathrm{eff}}}=0 .
$$

A general expression for any effective medium theory of a two-component composite is given by the spectral density theory. ${ }^{14}$ This theory states that the effective dielectric function can be expressed in the following integral representation:

$$
\varepsilon_{\mathrm{eff}}=\varepsilon_{m}\left[1-p \int_{0}^{1} \frac{g(u, p)}{t-u} d u\right],
$$

with $t=\varepsilon_{m} /\left(\varepsilon_{m}-\varepsilon_{i}\right), u$ is the integration variable and $g(u, p)$ is the so-called spectral density function. In short, this expression enables separating the role of geometry of the system [contained in $g(u, p)$ ] and the specific dielectric function of components $(t)$. It can be shown that when the inclusions present some degree of interconnection, the effective dielectric function reads as: $:^{15}$

$$
\varepsilon_{\mathrm{eff}}=p g_{0}(p) \varepsilon_{i}+\left[1-p g_{0}(p)\right] \varepsilon_{m}-\varepsilon_{i} p \int_{0}^{1} \frac{g_{\mathrm{cont}}(u, p)}{t-u} d u,
$$

where $g_{0}$ is the percolation strength and quantifies the degree of connection among inclusions and $g_{\text {cont }}$ accounts for the interaction among nonconnected inclusions. For a given composite geometry it is possible to calculate its spectral density function. ${ }^{16}$ Alternatively, if the effective dielectric function and the dielectric function of the components are known, the spectral density function can be numerically inferred. ${ }^{17,18}$

\section{Experimental}

A set of $\mathrm{SiO}_{2}-\mathrm{Ag}$ composistes was obtained by the subsequent deposition of $\mathrm{SiO}_{2} / \mathrm{Ag} / \mathrm{SiO}_{2}$ on $\mathrm{BK} 7$ substrates. $\mathrm{SiO}_{2}$ layers were $80-\mathrm{nm}$ thick and $\mathrm{Ag}$ layer mass thickness was $12 \mathrm{~nm}$. Due to the small amount of deposited metal, an island-like growth takes place resulting in the formation of metal clusters with nanometric dimensions, rather than a compact Ag film. ${ }^{19}$ The reason to surround $\mathrm{Ag}$ with $\mathrm{SiO}_{2}$ is to clearly identify the matrix where clusters are embedded. The depositions were performed by electron beam evaporation in a modified Varian chamber with a base pressure of $10^{-7}$ Torr, using quartz crystal to monitor the deposited nominal mass thickness. The deposition rates of $\mathrm{Ag}$ and $\mathrm{SiO}_{2}$ were $\sim 0.1$ and $1 \mathrm{~nm} / \mathrm{s}$, respectively. Deposition temperature, regulated with quartz heaters, was changed from sample to sample. It was varied from room temperature to $255^{\circ} \mathrm{C}$.

Optical measurements allowed retrieving the effective dielectric function and the effective thickness of the composite, in a similar way as described in Ref. 18. The measurements were taken in the spectral range between 275 and $2000 \mathrm{~nm}$ with a J. A. Woollam V-VASE ellipsometer. Ellipsometric data were taken at angles of incidence 45, 55, and $65 \mathrm{deg}$ and transmittance spectra were taken at normal incidence. This combination of measurements enables a precise simultaneous determination of the dielectric function and thickness of very thin absorbing films. ${ }^{20}$ It must be pointed out that the model used for the effective dielectric function was isotropic. In principle, one may expect that measurements at oblique incidence of a two-dimensional ensemble of nanoparticles will reveal different optical response owing the excitation of different plasmon resonance of particles depending on the polarization direction. ${ }^{21}$ Such anisotropy will 

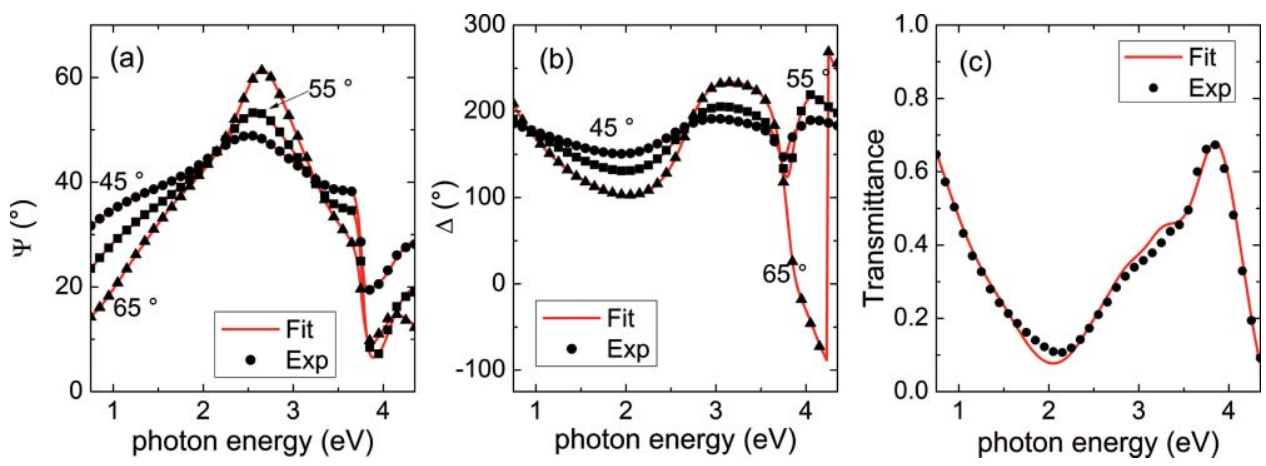

Fig. 1 Experimental data (dots) and model fits (solid line) for the sample deposited at $T=185^{\circ} \mathrm{C}$ : (a) and (b) are ellipsometric angles and (c) is transmittance spectrum.

be strongly enhanced if the particles have an elliptical shape. ${ }^{22}$ We did not observe such behavior in our samples. Indeed, the use of anisotropic models for the optical constants of the island film did not significantly improve the description of the data. We think that the width of the plasmon resonances excited parallel to the film plane is too large and overlaps the plasmon resonances excited perpendicular to the film plane.

\section{Results and Discussion}

\subsection{Choice of the Dispersion Model}

It was possible to obtain very good data fits using the multiple oscillator model described above for all the analyzed samples. Discrepancies between simulation and measurement were within the experimental error. Fits for ellipsometric angles $\Delta$ and $\psi$ and transmittance spectra for the sample that was obtained at deposition temperature $185^{\circ} \mathrm{C}$ are shown in Fig. 1. In this case, a Gaussian model was assumed for the oscillators representing the effective optical constants of the composite. If the Lorentz model is used, the obtained fits are significantly worse. In Fig. 2, the differences between the experimental measurements of the mentioned sample and the model simulations considering both Gaussian and Lorentz line shapes are shown. For all the samples, the best fits were obtained when using the Gaussian model. Thus, the $\chi^{2}$ estimator, quantifying the difference between the model simulation and experimental data, is typically 2 to 4 times higher if Lorentz line shapes are considered instead of Gaussian ones. Although the Lorentz model has been widely used for this type of composite, ${ }^{10,11}$ the Gaussian model is able to provide
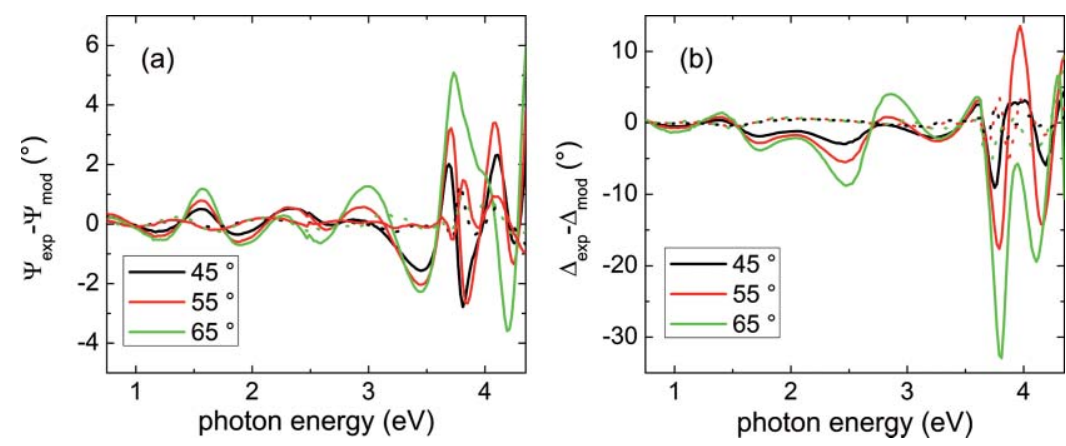

Fig. 2 Difference between experimental data and model fits for the ellipsometric measurements at different angles of incidence for the sample deposited at $T=185^{\circ} \mathrm{C}$ using either Lorentz (solid lines) or Gaussian oscillator models (dashed lines). 

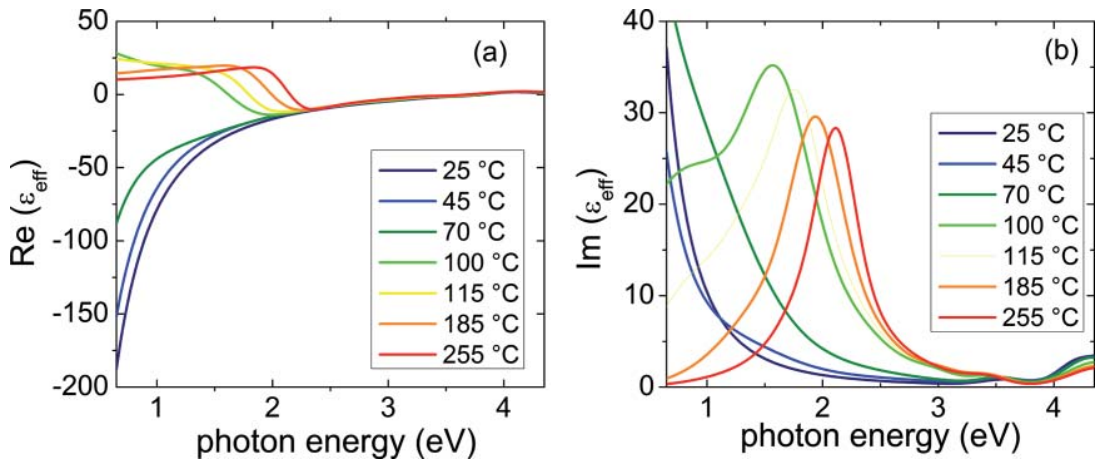

Fig. 3 Real (a) and imaginary (b) part of the effective dielectric function determined from experimental measurements for all the studied composites.

better fits, while having the same mathematical complexity, as the number of parameters of both models is the same.

\subsection{Effect of the Deposition Temperature on the Effective Dielectric Function}

The dispersion of the effective dielectric function for all the samples is shown in Fig. 3. It can be seen that at high deposition temperatures the optical constants are characterized by a narrow surface plasmon resonance that broadens and redshifts as the temperature decreases. At low temperatures the effective dielectric function follows the typical behavior of metals. These trends can be explained by the dependence of the island growth with the temperature: at high temperatures, nearly spherical and well isolated spherical particles are formed. ${ }^{23}$ The optical properties of such system are characterized by a narrow surface plasmon resonance. ${ }^{24}$ As the deposition temperature decreases, wetting of metal on dielectric improves and the island formation is prevented: ${ }^{23}$ the islands become more elliptical and closer to each other. Both of these effects results in broadening and a redshift of the surface plasmon resonance. ${ }^{24}$ At the lowest deposition temperatures the islands are able to percolate and the deposited metal forms a connected network, resulting in the observed metal-like optical behavior. These trends are supported by the evolution of the effective thickness of the composite that decreases from 33 (highest deposition temperature sample) to $13 \mathrm{~nm}$ (lowest deposition temperature sample), confirming the formation of a more compact layer as the deposition temperature decreases.

\subsection{Description of Results in the Framework of Effective Medium Theories}

According to the previous observations, one may expect that the samples deposited at higher temperatures are close to the assumptions of the Maxwell-Garnett theory while those samples deposited at lower temperatures should fit better into the Bruggeman description. Figure 4 compares the experimentally determined effective dielectric function with the computations of the classical effective medium theories, using as filling fraction the ratio between nominal and effective thickness and the optical constants for silver and silica taken from literature. ${ }^{25,26}$ There is some qualitative agreement between the results and the classical effective medium theories: the samples deposited at high temperature are characterized by the surface plasmon resonance while the samples deposited at lower temperatures have a metal-like behavior. However, quantitatively the differences are large, indicating that these classical theories cannot be used to model the behavior of these composites. Even if more advanced effective medium descriptions are considered, such as two-dimensional models or assumption of nonspherical shapes of inclusions, ${ }^{24}$ the experimental results still cannot be successfully described. 

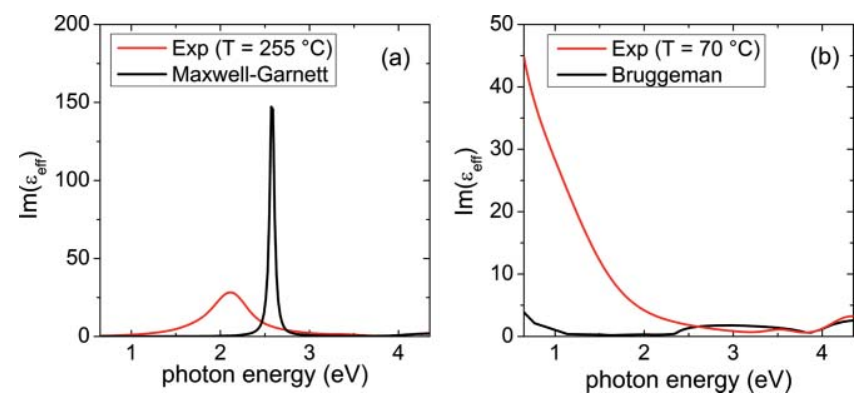

Fig. 4 Comparison between experimentally determined effective dielectric function (red lines) and the results of effective medium theories (black line) for samples obtained at high (a) and low (b) deposition temperatures.

The ability of classical effective medium theories to describe the composites deposited at intermediate temperatures is even worse, not only quantitatively but also from a qualitative point of view. For instance, these theories cannot describe the double-resonance structure appearing in the sample deposited at $100^{\circ} \mathrm{C}$, discussed below, and that has been observed recently in other metal-dielectric composites near the percolation threshold..$^{10}$ Similarly, these theories cannot account for the anomalous absorption observed in percolated systems such as nanoporous gold,${ }^{27}$ that has been recently explained on the basis of the localized surface plasmon resonance of a metal-core dielectric-shell particle embedded in a metal matrix. ${ }^{28,29}$ Finally, it must be highlighted that recent theoretical investigations ${ }^{30,31}$ suggest that these classical effective medium theories show nonplausible physical solutions when applied to systems consisting of nondissipative materials with real parts of their dielectric functions having opposite signs. Thus, it may be questionable whether these effective medium theories can be applied to metaldielectric composites, even when the composite geometry fulfills the requirements imposed by these theories.

In order to analyze whether it is possible to describe the experimentally determined effective dielectric function as a mixture of the component materials, we try to extract the spectral density function from the experimental measurements. Basically, Eq. (6) is numerically inverted imposing regularization conditions and assuming a discrete spectral density function. Details on the numerical procedure are given in Refs. 17 and 18. This procedure provides a spectral density function (Fig. 5) that satisfactorilly describes the effective dielectric function as a mixture of silver and silica for all the studied samples. The spectral density functions of samples deposited at higher deposition temperatures are characterized by a broad peak, that is in good agreement with the numerical simulations for random systems of spherical particles. ${ }^{16}$ As the
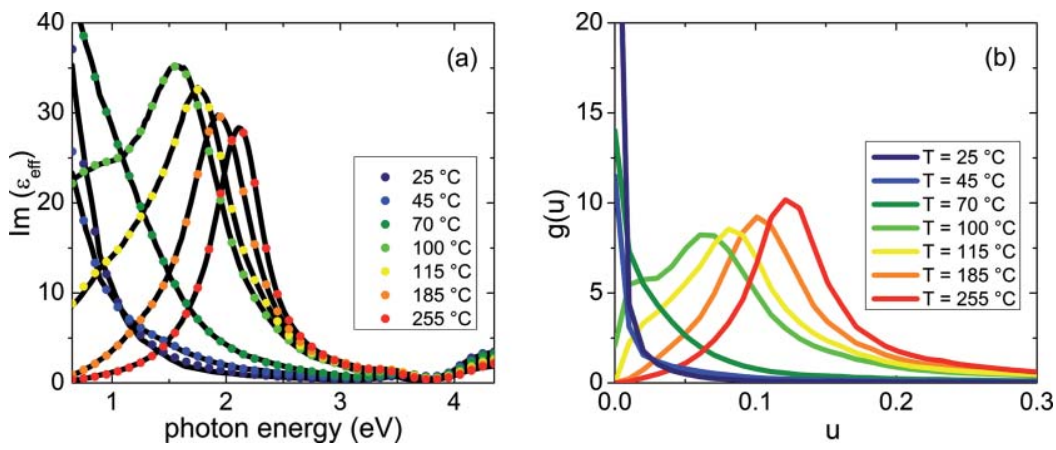

Fig. 5 (a) Experimentally determined effective dielectric function (dots) and fits obtained by numerical inversion of the spectral density function (solid lines). The corresponding spectral density functions are shown in (b). 
temperature becomes lower, the spectral density function acquires a multiple-peak structure. Such characteristic has been recently observed in other metal-dielectric composites ${ }^{32}$ and can be associated to different distributions of depolarization factors, corresponding to single islands and to large clusters of islands that start to appear as the temperature decreases. As pointed out in Ref. 32 such distribution of depolarization factors is more clearly revealed in the spectral density function than in the imaginary part of the dielectric function (only at the sample deposited at $100^{\circ} \mathrm{C}$ ). At low temperatures, the spectral density function presents the typical shape of fractal or percolated structures. ${ }^{15,18}$ The percolation strength, $g_{0}$, is zero for the samples deposited at temperatures higher than $70^{\circ} \mathrm{C}$, and increases as the deposition temperature decreases, in agreement with the observed metal-like behavior for samples deposited below $70^{\circ} \mathrm{C}$.

\section{Conclusions}

The results presented show that varying the deposition temperature only, it is possible to obtain a widely tunable effective dielectric function for metal-dielectric composites produced by e-beam evaporation. At high temperatures, the optical behavior is characterized by a narrow localized surface plasmon resonance while at low temperatures the composite presents a metallike behavior. Such broad tuning range of the dielectric function is very attractive from the application point of view as it enables large flexibility in the design of multilayer systems containing metal-dielectric composites.

The modeling based on the multiple oscillator approach reveals that assuming a Gaussian line shape for the absorption of the composite can provide a more accurate description of the experimental data than using the classical Lorentz oscillator model.

Finally, the description of the experimentally determined effective dielectric function in terms of simple classical effective medium theories fails. Nevertheless, the composites can be successfully described as mixtures of the composing materials if the Bergman spectral density representation is used. In this case, spectral density functions that are in good agreement with previous numerical simulations are found.

\section{References}

1. S. A. Maier, Plasmonics: Fundamentals and Applications, Springer, New York (2007).

2. W. Cai and V. Shalaev, Optical Metamaterials: Fundamentals and Applications, Springer, New York (2009).

3. S. Kachan, O. Stenzel, and A. Ponyavina, "High-absorbing gradient multilayer coatings with silver nanoparticles," Appl. Phys. B 84, 281-287 (2006).

4. V. Janicki, J. Sancho-Parramon, and H. Zorc, "Gradient silver nanoparticle layers in absorbing coatings - experimental study," Appl. Opt. 50, C228-C231 (2011).

5. H. Zorc, M. Loncaric, and J. Sancho-Parramon, "Use of gold island films in design of reflectors with high luminosity," Appl. Opt. 50, C364-C367 (2011).

6. V. Janicki, J. Sancho-Parramon, F. Peiró, and J. Arbiol, "Three-dimensional photonic microstructures produced by electric field assisted dissolution of metal nanoclusters in multilayer stacks," Appl. Phys. B 98, 93-98 (2010).

7. V. Janicki, J. Sancho-Parramon, and H. Zorc, "Multilayer based interferential-plasmonic structure: metal cluster 3D grating combined with dielectric mirror," Appl. Phys. A (accepted for publication).

8. C. F. Bohren and D. R. Huffman, Absorption and Scattering of Light by Small Particles, Wiley, New York (1983).

9. J. Sancho-Parramon, "Surface plasmon resonance broadening of metallic particles in the quasi-static approximation: Numerical study of size confinement and interparticle interaction effects," Nanotechnology 20, 235706 (2009). 
10. M. Hövel, B. Gompf, and M. Dressel, "Dielectric properties of ultrathin metal films around the percolation threshold," Phys. Rev. B 81, 035402 (2010).

11. A. J. de Vries, E. S. Kooij, H. Wormeester, A. A. Mewe, and B. Poelsema, "Ellipsometric study of percolation in electroless deposited silver films," J. Appl. Phys. 101, 053703 (2007).

12. WVASE manual "Guide to Using WVASE32," J. A. Woollam Co., Inc.

13. S. Bosch, J. Ferré-Borrull, N. Leinfellner, and A. Canillas, "Effective dielectric function of mixtures of three or more materials: A numerical procedure for computations," Surf. Sci. 453, 9-17 (2000).

14. D. J. Bergman, "The dielectric constant of a composite material-A problem in classical physics," Phys. Rep. 43, 377-407 (1978).

15. W. Theiss, "The use of effective medium theories in optical spectroscopy," Adv. Solid State Phys. 33, 149-176 (1994).

16. K. Hinsen and B. U. Felderhof, "Dielectric constant of a suspension of uniform spheres," Phys. Rev. B 46, 12955-12963 (1992).

17. A. R. Day, A. R. Grant, A. J. Sievers, and M. F. Thorpe, "Spectral function of composites from reflectivity measurements," Phys. Rev. Lett. 84, 1978-1981 (2000).

18. J. Sancho-Parramon, V. Janicki, and H. Zorc, "On the dielectric function tuning of random metal-dielectric nanocomposites for metamaterial applications," Opt. Express 18, 2691526928 (2010).

19. M. Loncaric, J. Sancho-Parramon, M. Pavlovic, H. Zorc, P. Dubcek, A. Turkovic, S. Bernstorff, G. Jakopic, and A. Haase, "Optical and structural characterization of silver islands films on glass substrates," Vacuum 84, 188-192 (2009).

20. J. N. Hilfiker, N. Singh, T. Tiwald, D. Convey, S. M. Smith, J. H. Baker, and H. G. Tompkins, "Survey of methods to characterize thin absorbing films with Spectroscopic Ellipsometry," Thin Solid Films 516, 7979-7989 (2008).

21. R. G. Barrera, M. del Castillo-Mussot, G. Monsivais, P. Villasenor, and W. L. Mochán, "Optical properties of two-dimensional disordered systems on a substrate," Phys. Rev. B 43, 13819-13826 (1991).

22. T. Wenzel, J. Bosbach, A. Goldmann, F. Stietz, and F. Träger, "Shaping nanoparticles and their optical spectra with photons," Appl. Phys. B 69, 513-517 (1999).

23. N. Kaiser, "Review of the fundamentals of thin-film growth," Appl. Opt. 41, 3053-3060 (2002).

24. U. Kreibig and M. Vollmer, Optical Properties of Metal Clusters, Springer-Verlag, New York (1995).

25. E. Palik, Handbook of Optical Constants of Solids, Academic Press, New York (1997).

26. P. B. Johnson and R. W. Christy, "Optical constants of the noble metals," Phys. Rev. B 6, 4370-4379 (1972).

27. A. I. Maaroof, A. Gentle, G. B. Smith, and M. B. Cortie, "Bulk and surface plasmons in highly nanoporous gold films," J. Phys. D: Appl. Phys. 40, 5675-5682 (2007).

28. G. B. Smith and A. A. Earp, "Metal-in-metal localized surface plasmon resonance," Nanotechnology 21, 015203 (2010).

29. A. A. Earp and G. B. Smith, "Metal nanoparticle plasmonics inside reflecting metal films," Appl. Phys. Lett. 96, 243108 (2010).

30. T. G. Mackay, "On the effective permittivity of silver-insulator nanocomposites," $J$. Nanophoton. 1, 019501 (2007).

31. A. Duncan, T. G. Mackay, and A. Lakhtakia, "On the Bergman Milton bounds for the homogenization of dielectric composite materials," Opt. Commun. 271, 470-474 (2007).

32. E. Tuncer and G. A. Niklasson, "Optical properties of non-dilute metal insulator composites," Opt. Commun. 281, 4374-4379 (2008).

Biographies and photographs of the authors not available. 